\title{
Internet-Based Technologies for Design of Embedded
}

\section{Systems}

\author{
Anzhelika Parkhomenko, Olga Gladkova, Sergey Kurson, Aleksandr Sokolyanskii and Eugene Ivanov \\ Software Tools Department, Zaporizhzhya National Technical University, Zaporizhzhya 69063, Ukraine
}

\begin{abstract}
In recent years, online engineering technologies are widely distributed and developed. Their influence on society is very strong. The Internet technology has provided additional opportunities for a new development level of education, design and production. Associations and scientific conferences in the field of online engineering that appeared, seek to foster practices in education and research in higher education institutions and the industry on online engineering. A particular challenge for online engineering is how to extend the traditional equipments and laboratories to the Internet. A method of the embedded systems design with using online laboratory is described in this paper. Also, in this paper the experimental set of remote laboratory which allows carrying out hardware/software oriented design of the embedded control system of a mobile platform is considered.
\end{abstract}

Key words: Online engineering, Internet technology, remote laboratory, embedded system design, experimental set, hardware and software components.

\section{Introduction}

The mushroom growth of Internet technologies and its increasing popularity has had an enormous impact on engineering. The shared use of virtual and remote controlled devices as well as distributed remote and virtual laboratories is an actual task today, due to:

- The growing complexity of engineering tasks;

- More and more specialized and expensive equipment as well as software tools and simulators;

- The necessity of use of expensive equipment and software tools/simulators in short time projects;

- The application of high tech equipment also in SMEs (small and medium-sized enterprises);

- The need of high qualified staff to control recent equipment;

- The demands of globalization and division of labor [1].

Thus it is increasingly necessary to allow and organize a shared use of equipment, as well as specialized software, for example simulators.

Corresponding author: Anzhelika Parkhomenko, Ph.D., Docent, research fields: CAD/CAM/CAE systems, embedded systems of mobile objects control, technologies and systems of virtual and remote engineering. E-mail: parhom@zntu.edu.ua.
Due to the above mentioned, created and successfully function various remote and virtual laboratories. In the first case we are dealing with the so-called laboratory installation with remote access, which includes real lab, hardware and software for plant control and digitization of the received data, as well as means of communication. In the second case, all processes are modeled using the computer.

Virtual laboratories are a wider notion, which concern two types of hardware-software complexes:

- Laboratory installation with remote access - they are called such a set of RL (Remote Laboratory);

- Software that allows you to simulate laboratory experiments-Virtual Laboratory (in the narrow sense).

Today RLs are successfully developed and implemented worldwide and give opportunity to remotely use the equipment, without need to buy and establish it in place. Such laboratories provide access to the experiments in the fields of electronics, physics, chemistry, biology, mechanics, earth sciences, etc. They are usually used for educational purposes for pupils and students. The numbers of such laboratories keep growing, though it is not easy to create an 
interface for remote experiments, solve the task of handling and safety.

But the problem of sharing use of the equipment in the production and research purposes, as well as realization of remote experiments for rapid prototyping of designed objects remains relevant.

The aim of this work is to study and prove in practice the capabilities of creation and using of remote laboratories for embedded systems design.

\section{Fields of Application of Virtual and Remote Laboratories}

Remote Laboratory is a hardware-software complex, allowing to hold research without direct contact with the actual installation or its complete absence. The first concepts of laboratories of remote access started appearing in 1991. By 2006 one third of all realized projects with remote access was represented by experiments from physics area. While engineering and robotic projects make two-thirds of all laboratories of remote access [2]. Today more and more widely used of remote (virtual) laboratories in education extends that allows to lower significantly costs of creation and the maintenance of a practical work for training of specialists of technical specialties [3, 4].

In the industry laboratory of remote access can be applied where direct contact of the person with devices and equipments which are intended for investigation of object for a number of reasons it is impossible [5].

We carried out research of architectural and functional features of a number of remote laboratories and below are some examples of virtual laboratories for the field of control engineering, robotics, tele-control engineering for industry and education. One of the first was analyzed remote laboratory GOLDI (Grid of Online Lab Devices Ilmenau) of Technical University of Ilmenau (Germany), access to which we got within the framework of international ICo-op TEMPUS project [6].

Hybrid online laboratory GOLDI-was developed at the Department of Integrated Communication
Systems at the Ilmenau University of Technology. It provides a tool set, supporting all design steps for complex control tasks (e.g., in the fields of control engineering, robotics, tele-control engineering) [7].

WebLab-Deusto - a remote laboratory for technical curricula, provided by the Faculty of Engineering of the University of Deusto. It can be downloaded and deployed to serve new remote experiments in different environments and operating systems [8].

WEBENCH ${ }^{\circledR}$ Design Center-virtual laboratory of Texas Instruments. Design tools WEBENCH represent effective software implemented algorithms and graphical user interfaces that provide fast work of applications for power management, developing drivers for powering LEDs (light-emitting diodes), and applications that work with different sensors (measuring devices). These easy-to-use tools help you create, optimize and simulate designs that meet the unique technical requirements of development. This allows the user to compare the current cost on system level and supply chain before design [9].

Labshare-Australia national nonprofit network of laboratories with the remote access. Includes five technical universities. Experimental stands allow to solve various problems (physics, control engineering, robotics, tele-control engineering). Comprises ten experiments [10].

e-Laboratory Project - the remote laboratory of the Czech Republic and Slovakia providing access to ten experiments in the fields of electrical engineering and physics [11].

iLabs - the online laboratory, created for enrichment of laboratory practical works in engineering education. Remote lab is created at the Massachusetts Institute of Technology. Totals about twenty experiments in the field of microelectronics, chemical industry, polymer crystallization, design of constructions and processing of signals [12].

Also, the laboratories and many others were analyzed [13-15].

The analysis of the existing laboratories showed: 
- The main directions of development of remote and virtual engineering today are in the field of electronic equipment and robotics;

- The problem is that the majority of experiments and installations of remote access represent more educational resources and that the possibilities for the professional researcher or designer are not fully explored.

\section{Basic Concept for Remote-Based Embedded Systems Design}

Today the market of ES (Embedded Systems) is permanently evolving and requires the creation of more complex systems in shortening periods of time. Under these conditions, the development of systems "from scratch" is not effective. Therefore, one of the key areas to improve the efficiency of ES design, is the accumulation of technical solutions for reuse. The disunity of descriptions of already developed components (hardware units, programs, algorithms, implementations, etc.) hinders their reuse, but the application of remote experiments will allow the designer to obtain information for making decisions on the ES realization.

On the other hand, on a step of hardware and software integration there are some problems too. For observance of terms of the embedded system integration it is necessary to begin work with an incomplete set of ready devices since readiness of various devices comes in various time.

As indicated in Ref. [16], in classical ES design the concurrent process of hardware/software components design is distributed in the timeline (Fig. 1). The established practices of ES design show that hardware decisions are initially selected. Further, based on it the software superstructure will be made. In the worst case, the firmware and software developer can therefore not start to develop and test their software until the hardware design is available. This also has to great risk of delaying the whole product design chain.

That is why in the field of ES design is very popular use of ready hardware/software platforms allowing to accelerate development of products so to reduce the time to market. Researches had shown there are many different manufacturers of hardware/software platforms: Arduino, Texas Instruments, Parallax Inc., Netmedia, Microchip, Digilent, MBED, Raspberry PI, Cyclone and others. Each platform has the own form factor and functionality, and its choice depends on the solved task. For developers of the ES an assessment and a choice of suitable solutions become more and more actual because of continuous growth of the

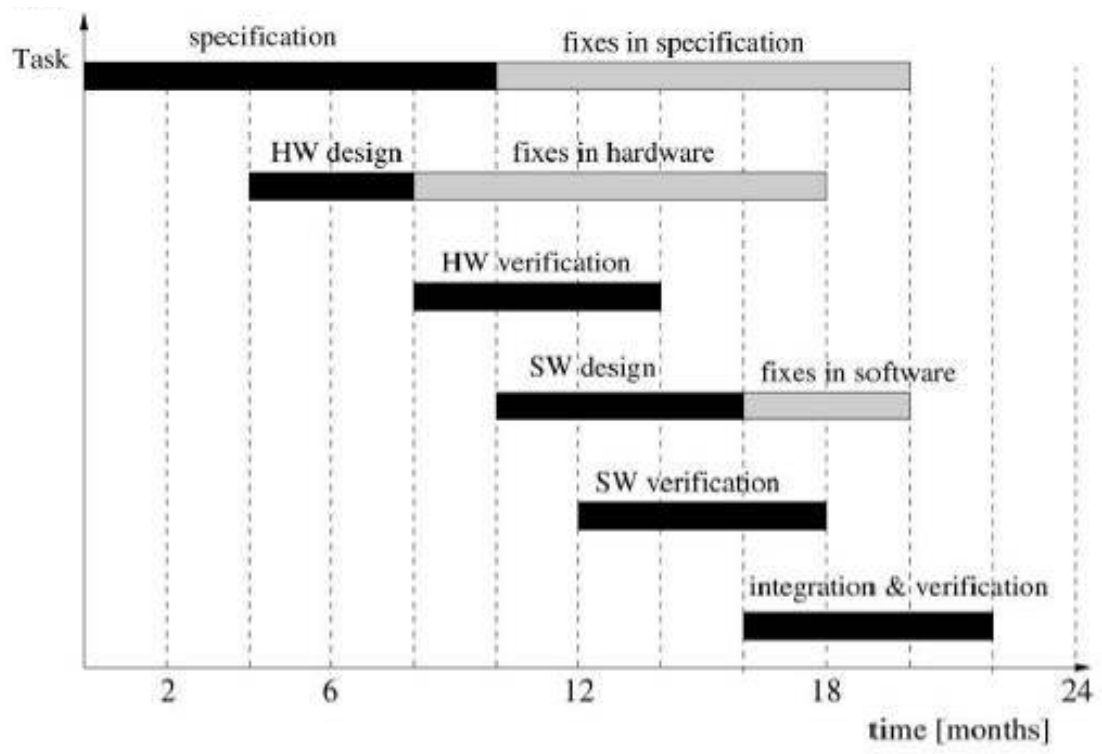

Fig. 1 Classical embedded systems evolution-Timeline. 
nomenclature of component base and expansion of a scope of the ESs.

Thus, the designer of ES has to know possibilities of the ready platforms existing in the market and be able to make the responsible decision concerning application of a certain hardware-software platform. Unfortunately, the information which is offered on the website not always allows to make the right reasonable choice and optimally implement the project. Acquisition of a huge number of various platforms by small and medium-sized enterprises is unacceptable for the analysis of their opportunities, despite the relatively low prices [17].

Due to growth of popularity of ready hardware-software platforms, a number of the companies offer applications for virtual simulation of their work (for example, free simulator 123D Circuits from Autodesk) [18].

However, the simulation does not replace real work with the hardware and software. Modeling accuracy is determined by the implemented mathematical model. Besides that, many software simulators are not free, require time for their studying, have limited functionality and incomplete element base, the use of their full functionality is not always required, and the cost is quite high.

Therefore, creation and practical application of specialized laboratory for research of ready hardware-software platforms on the basis of remote experiment is an actual task.

The conception of using the remote laboratory for ES design presented in Ref. [19]. Using capabilities of remote lab, you can more optimally organize the project and realize it in a shorter time. A timing diagram of the project with using a remote lab can be changed, as shown in Fig. 2 [16]. Compared with the Timeline in Fig. 1, the time-to-market of products reduced and released time for research and development of innovative solutions.

\section{Client-Server Technologies of Remote Laboratory Development}

At the stage of selecting of Web Service creation technology has been carried out a comparative analysis of the main server programming platforms: ASP.NET, JAVA, PHP. Comparison was carried out according to several criteria.

PHP is an interpreted language that allows to mix code and HTML in a single file. Java and ASP.NET

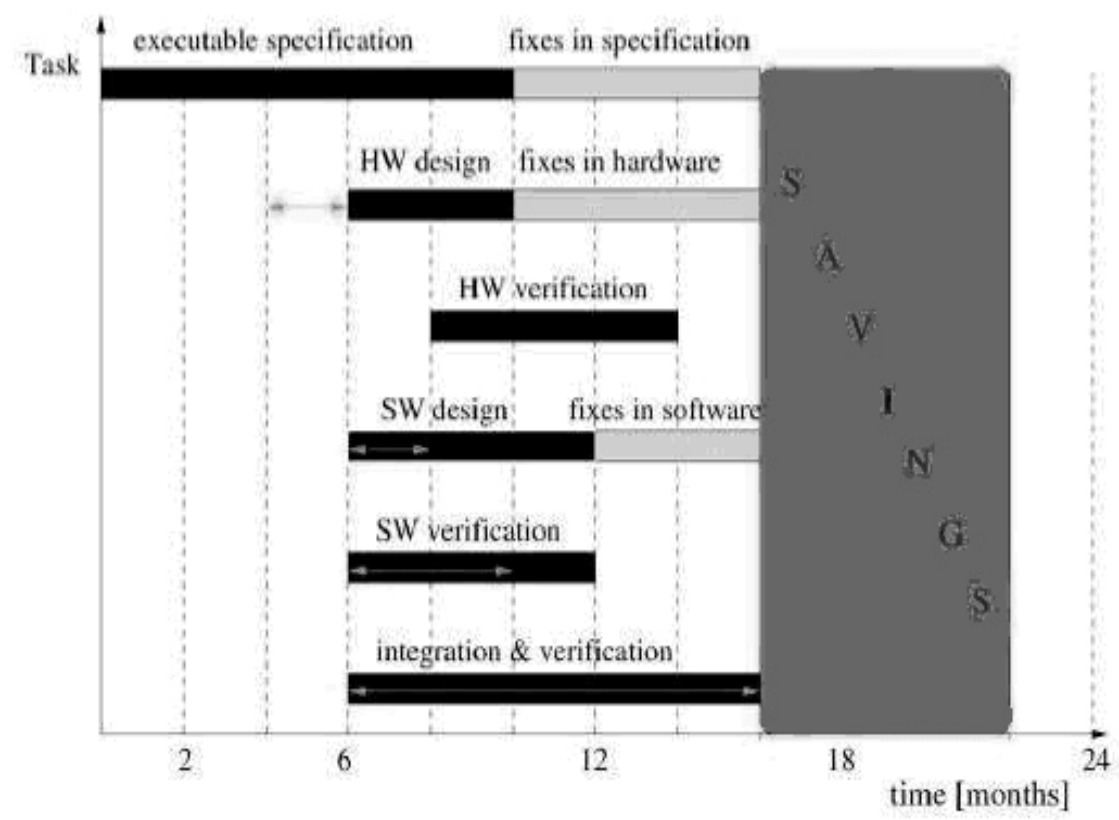

Fig. 2 Design flow allowing concurrent development of hardware and software. 
dynamically compiled when the page is requested. MySQL for PHP, Oracle for Java, and MSSQL for ASP.NET can be applied for the simplest use of the database. For ASP.NET must be used with the Windows platform or Microsoft IIS Server, while JAVA and PHP can run on systems like UNIX and Windows. Eventually PHP was chosen because it is the most cost-effective solution. Software, database, operating system is free of charge.

After selecting a server language, it has been selected PHP MVC framework CodeIgniter to improve the quality of development and systematization code. The MVC pattern describes a simple method for constructing the structure of an application, the aim of which is to separate the business logic from the user interface. As a result, the application is easier developed, scaled, tested and accompanied.

Framework CodeIgniter main advantages are: it is almost micro framework; it is very easy to learn; excellent documentation; it is flexible; allows easily use third-party code.

For HTML adaptive typesetting has been selected Bootstrap which includes HTML and CSS web pages templates. JavaScript (jQuery) is used for data processing on the client side.

For laboratory the several controllers were created using MVC framework CodeIgniter which include all the laboratory logic. Also was created several views to display web-pages with data.

Three options of video broadcast remote experiments were considered:

- HTTP-stream + tag < video $>$ to display.

- Motion JPEG HTTP-stream + tag $<$ img $>$ to display.

- MPEG-stream through WebSockets + tag $<$ canvas $>$ to render.

The first option is: via VLC player will be captured video from the camera and broadcasts it to the HTTP-protocol. Tag < video $>$ captures video at a specific address and play it. Advantages: simplicity of implementation. Disadvantages: navigation and control tools available in the tag $<$ video $>$ in this case are not necessary and may hinder; videos caching; high delay.

On the server side of the second option is the same as the first except for the broadcast. On the client side for the displaying is a tag <img>, which is constantly updated source of the image, giving the impression of a video. Advantages: simplicity of implementation; no video caching; low delay. Disadvantages: loading channel and high traffic; inadequate smoothness; it is not a video, but the sequence of images.

The third option is a complete video transmission. This option does not have disadvantages of previous versions and have the advantage of them. Broadcast performed by ffmpeg. Through web sockets in JavaScript obtain the frames. Using jsmpeg (MPEG1 video decoder) decode them. After decoding the frame is drawn in the tag < canvas> through WebGL. Advantages: no video caching; low delay; full-motion video; smoothness; smaller loading channel and traffic. Disadvantages: more complex implementation.

So, for development client-server side laboratory was chosen the next technologies: for creation Web Service-PHP server programming languages, HTML and CSS; for video broadcast-MPEG-stream through WebSockets + tag $<$ canvas $>$ to render.

With constantly changing system requirements, the project can be difficult when using PHP and CodeIgniter, so it can be moved to another server platform, for example, JAVA.

The architecture MVC will allow without difficulty to import our system to another platform.

\section{Architecture of the Remote Lab}

RELDES (Remote Laboratory for Design of Embedded Systems) was developed to facilitate the remote communication between the experimenter of the embedded systems and the experimental device. It is friendly to users also for non-programmers who can easily design a variety of remote experiments. RELDES will allow the designer to obtain information about ready hardware and software platforms and 
components for making decisions on the ES realization.

The schematic view of the RELDES architecture is shown in Fig. 3 and main page interface in Fig. 4.

The set of experiments depends on specifics of the designed ES. For example, as standard decisions for design of mobile objects control system, experiments based on Arduino boards (Uno and Mega) as well as the servos, the liquid crystal display, the LEDs, the distance sensor, etc. are offered. The proposed set of experiments can be expanded for other hardware-software platforms, and tasks connected with realization of various classes of $\mathrm{ES}$.

The stand with experiments is connected to the laboratory server via the serial interface. The computer with Linux (Debian/Ubuntu) OS acts as the server of remote laboratory. The server provides access to experiment programming and video stream of the laboratory. Video stream is implemented with use of "ffmpeg". The server processes web client requests and carries out the following actions depending on the obtained data:

- Compilation of received initial code (Arduino, with use of the console utility Ino).

- Returning the compilation results (using HTTP protocol, as well as the sends request for compilation).

- Uploading binaries to the microcontroller (provided that the controller is free).

- Client queuing (if experiment is occupied).
The RELDES activity diagram is shown in Fig. 5. To get access to the experiments user needs to be registered. Access to the experiments page is closed for unregistered users. Users can upload their program code to a microcontroller on the Arduino board via the Web-client. With using of the Web-interface the user is able to create own code or upload file with initial code. The Lab server compiles received initial code. Being compiled, the generated code is uploaded to the microcontroller on the Arduino board. If the program has errors, the server generates and returns the compilation results.

Therefore, the user has the possibility to create and edit their program code directly in the client's Web interface without any need in development tools on the user's PC.

Client-server communication is carried out via web-interface implemented with $\mathrm{HTML}+\mathrm{CSS}+\mathrm{JS}$. The service is running on the Apache server with use of the relational MySQL database.

Fig. 6 illustrates an impression of the experiment page Web-interface. The user friendly slider for fast switching between experiments is located at the top of the page. The remaining area is divided for the displaying video and code editing. At start of work with remote experiment user can observe time remained to the end of experiment. Besides web browser no additional software is required to access the remote laboratory.

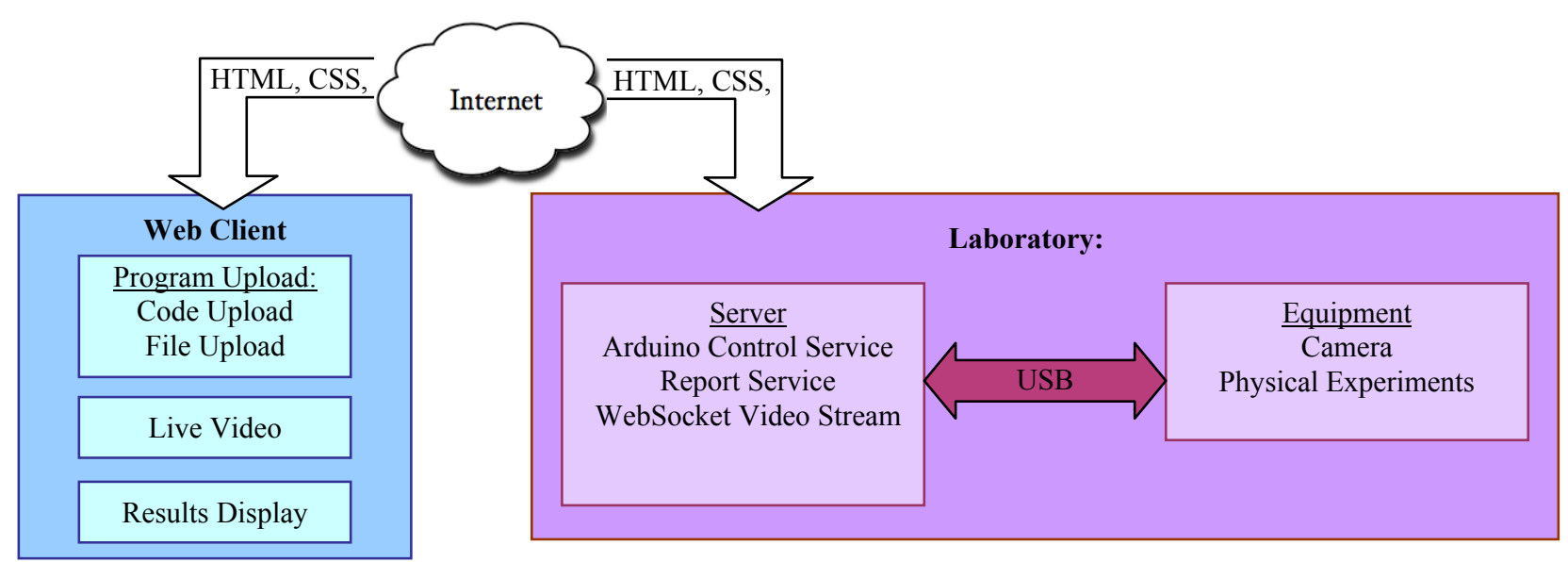

Fig. 3 Laboratory architecture. 


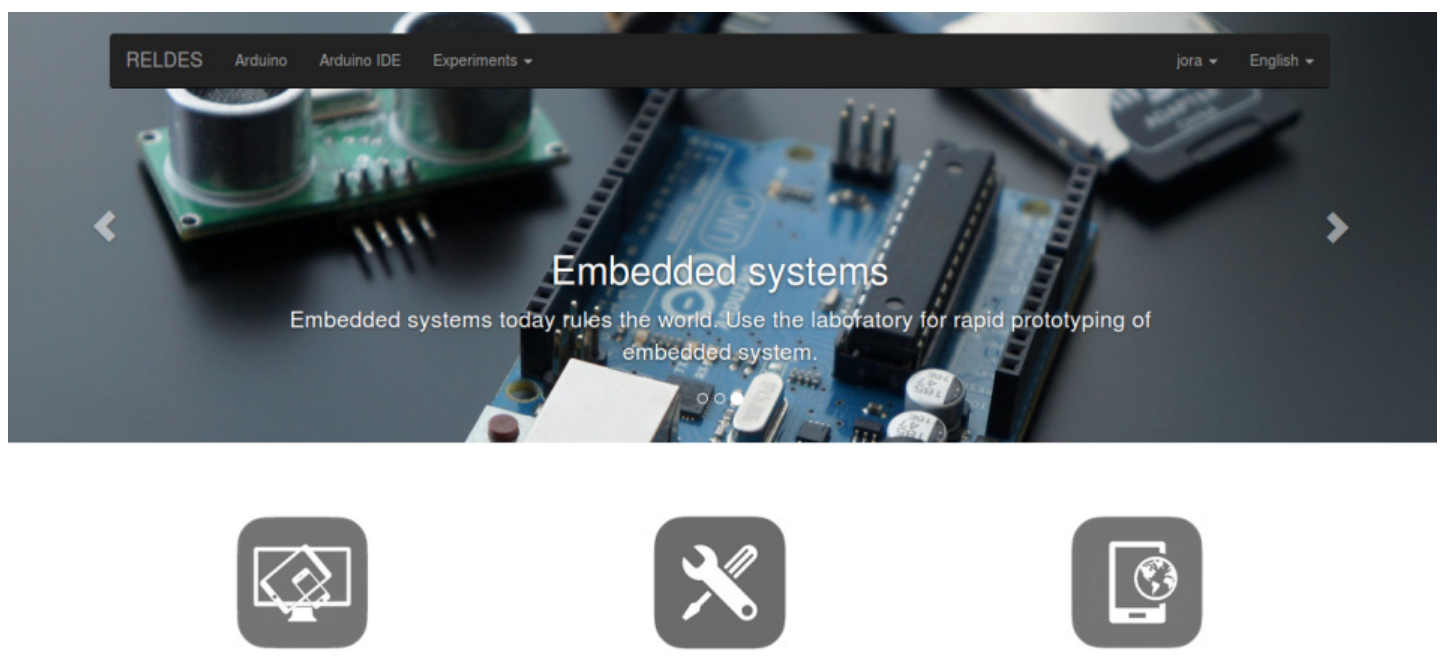

Fig. 4 Laboratory main page interface.

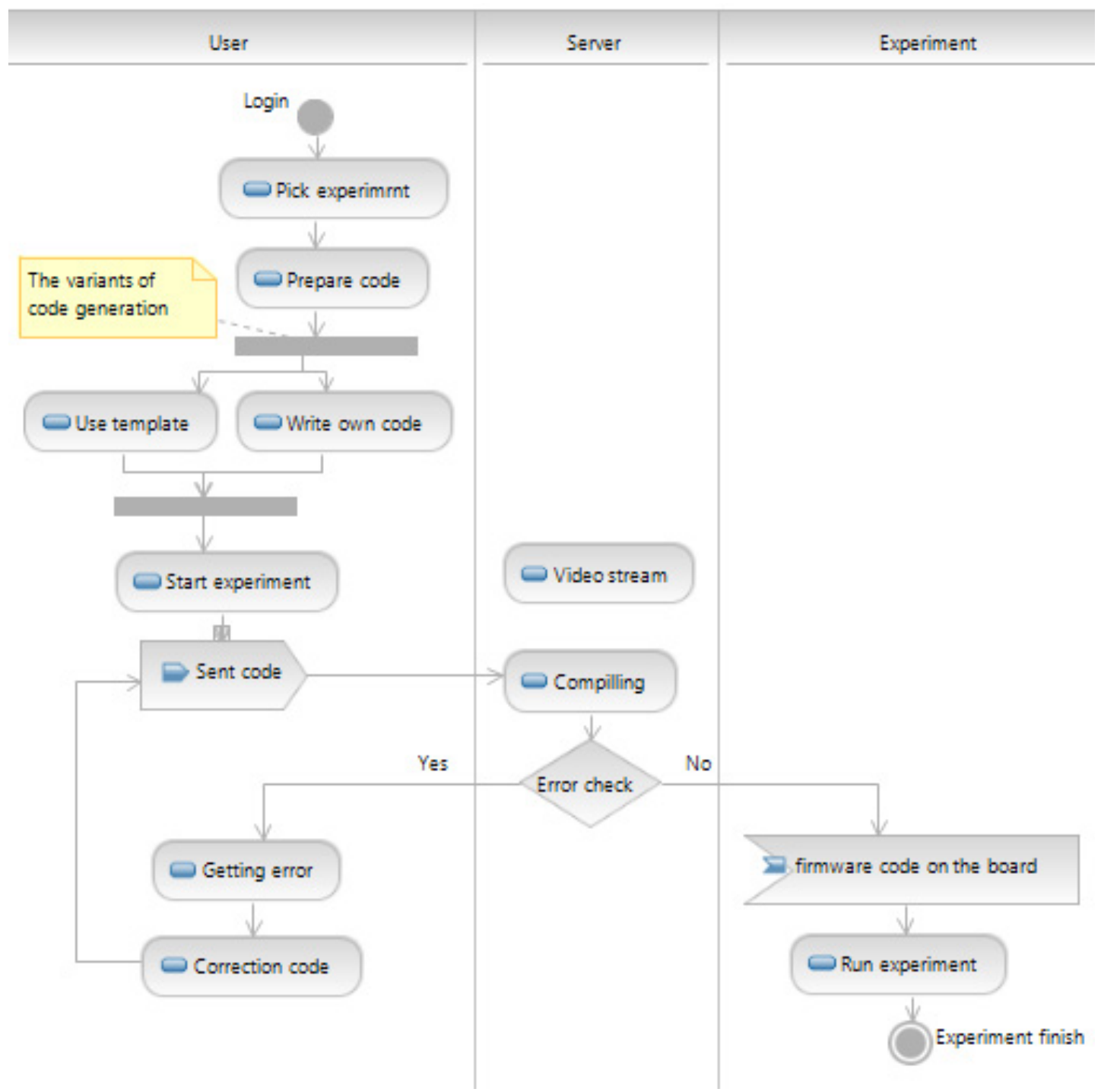

Fig. 5 Laboratory activity diagram. 


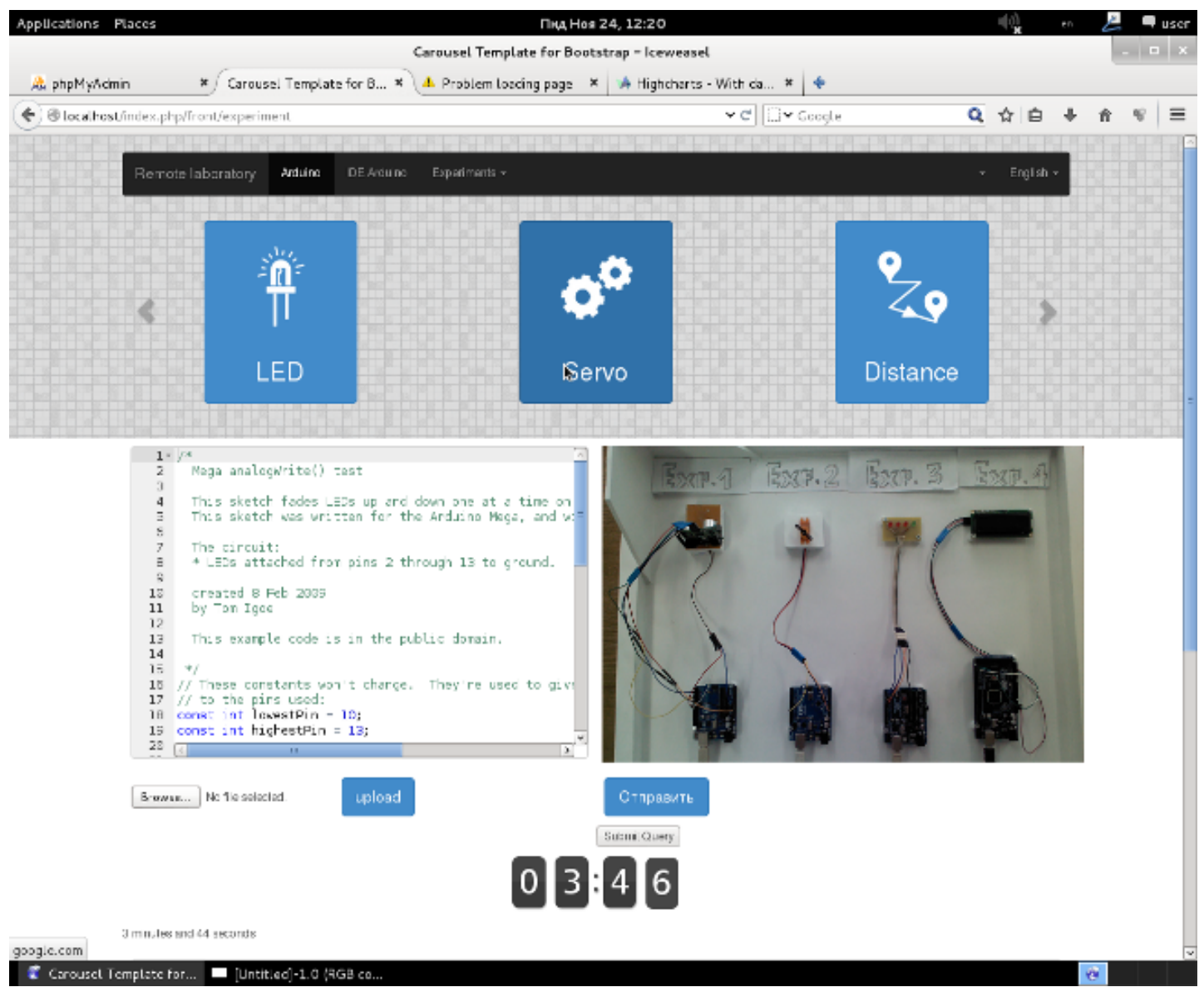

Fig. 6 Laboratory experiment page interface.

\section{Results and Discussion}

In this work the remote laboratory RELDES designed at the Software Tools Department, Zaporizhzhya National Technical University, Ukraine is described. Benefits of our laboratory:

- Cross-platform (use any device and operation system).

- Accessibility (use our hardware instead of spending money).

- Configurability (write your own code or use code templates).

The application of remote lab for ES design helps to solve the next tasks: information search and choosing of ready hardware/software platforms; providing developer access to testing of the equipment without worrying about its purchase or development; integration of hardware and software solutions; the creation and test of a rapid prototype of the future product.
Laboratory has been successfully applied in the implementation of the project of the embedded control system of a mobile platform [20].

\section{Conclusion}

The development of Internet technology has had an enormous impact on engineering. This has contributed to the development of important areas of researches such as remote and virtual engineering. Emergence of remote laboratories allows solving such problems as: sharing of the equipment; save time and money, released time for research and development of innovative solutions, etc.

But to achieve these goals need to create a laboratory not only for education, but also for professional researchers and designers.

Practical application of the developed RELDES laboratory will allow the designer to make the right decision choice on expediency of application of ready 
platform, and possible options of the project development in the field of ES design.

In the nearest future for the improvement of our laboratory we are implementing a mechanism of prior reservation time for carrying out experiments for enhancing the efficiency of users.

\section{Acknowledgement}

The authors would like to thank Prof. K. Henke and H.-D. Wuttke from Ilmenau University of Technology, Ilmenau, Germany for their support within the laboratory GOLDI [7].

This work is also supported by the project "ICo-op Industrial Cooperation and Creative Engineering Education based on Remote Engineering and Virtual Instrumentation" by the European Commission within the program "Tempus", Grant No. 530278TEMPUS-1-2012-1-DE-TEMPUS-JPHES [6].

\section{References}

[1] International Association of Online Engineering. $\mathrm{http} / / /$ online-engineering.org/index.php?option=com_con tent $\&$ view $=$ article $\&$ id $=53 \&$ Itemid $=68$.

[2] Chernyi, O. P., and Lashko, I. V. 2013. "Features of the Process of Preparation of Engineering Specialties." Engineering and Educational Technology in Electrical and Computer Systems (2): 9-19.

[3] Henke, K., Tabushchyk, G., Wuttke, H. D., Ostendorff, St., and Vietzke, T. 2014. "Using Interactive Hybrid Online Labs for Rapid Prototyping of Digital Systems." International Journal of Online Engineering 10 (5): 57-62.

[4] Henke, K., Wuttke, H. D., Ostendorff, St., Vietzke, T., and Lutze, C. 2013. "Fields of Applications for Hybrid Online Labs." International Journal of Online Engineering 9 (5): 20-30.

[5] Postnykov, E. B. 2011. "Review of World Experience of Creation and Operation of Laboratories of Remote
Access." http:www.efmsb.ru/download.

[6] ICo-op Project Website: Industrial Cooperation and Creative Engineering Education Based on Remote Engineering and Virtual Instrumentation 530278-TEMPUS-1-2012-1-DE-TEMPUS-JPHES. http://www.ico-op.eu/.

[7] The Grid of Online Laboratory Devices Ilmenau. http://www.tu-ilmenau.de/goldi.

[8] WebLab-Deusto. https://www.weblab.deusto.es/web.

[9] WEBENCH ${ }^{\circledR}$ Design Center. http://www.ti.com/lsds/ti/ analog/webench/overview.page.

[10] Remote Labs. Enriching Digital Education. http://www.labshare.edu.au/.

[11] e-Laboratory Project. http://www.ises.info/ index.php/en/.

[12] The iLab Project. https://wikis.mit.edu/confluence/ display/ILAB2/Home;jsessionid=F02FCB44122201A3D FB256BE892B9CDA.

[13] Remote-LAB GymKT. http://remote-lab.fyzika.net/ vzdalene-experimenty.php?lng=en\#DERIL.

[14] Remotely Controlled Experiments. http://ictphysics.upol. cz/remotelab/rlab_en.html.

[15] OpenLabs Electronics Laboratory. http://openlabs.bth.se/ electronics/index.php/en.

[16] Teich, J. 2012. "Hardware/Software Codesign: The Past, the Present, and Predicting the Future." IEEE Institute of Electrical and Electronics Engineers Journal (Special Centennial Issue) 100: 1411-29.

[17] Parkhomenko, A., and Gladkova, O. 2014. "Interactive Remote Laboratory for Research of Hardware-Software Platforms." In Proceedings of the International Scientific-Practical Conference Internet-Education -Science, 111-3.

[18] Electronics from Beginner to Pro. http://123d.circuits.io/.

[19] Parkhomenko, A., Gladkova, O., Ivanov, E., Sokolyanskii, A., and Kurson, S. 2015. "Development and Application of Remote Laboratory for Embedded Systems Design." International Journal of Online Engineering 11 (3): 27-31.

[20] Parkhomenko, A., and Gladkova, O. 2014. "Virtual Tools and Collaborative Working Environment in Embedded System Design.” In Proceedings of the 11th International Conference on Remote Engineering and Virtual Instrumentation, 91-3. 